Ingeniería Ecológica

\title{
Efecto de la concentración de metales pesados en la respuesta fisiológica y capacidad de acumulación de metales de tres especies vegetales tropicales empleadas en la fitorremediación de lixiviados provenientes de rellenos sanitarios.
}

ECOLOGICAL ENGINEERING

\section{Effect of heavy metal concentration on the physiological responses and heavy metal accumulation of three tropical plant species used for phytoremediation of landfill leachate}

\author{
Carlos A Madera-Parra*§, Enrique J Peña-Salamanca**, Juliana A Solarte-Soto**. \\ * Escuela Eidenar Facultad de Ingeniería, Universidad del Valle. Cali, Colombia. \\ **Departamento de Biología, Facultad de Ciencias Naturales y Exactas, Universidad del Valle. Cali, Colombia. \\ carlos.a.madera@correounivalle.edu.co,enrique.pena@correounivalle.edu.co,jass0420@hotmail.com.
}

Recibido: 10 de Mayo de 2013 - Aceptado: 1 de Junio de 2014

\begin{abstract}
Resumen
Este estudio evaluó el efecto de las concentraciones de los metales pesados (MP) $\mathrm{Hg}^{+2}, \mathrm{Cd}^{+2}, \mathrm{Cr}^{+6}, \mathrm{~Pb}^{+2}$, en la respuesta fisiológica y acumulacion de MP de las especies: Colocasia esculenta (Ce), Heliconia psittacorum (He) y Gynerium sagittatum (Gs), sembradas en humedales subsuperficiales de flujo horizontal (HCFSH) para el tratamiento de lixiviados de relleno sanitario (LX). Se tuvo un diseño factorial con 16 reactores operados en dos bloques experimentales. Clorofila, potencial hidrico y concentracion de MP fueron medidos en tejidos de las plantas. Diferencias significativas $(p<0,05)$ en los valores de clorofila entre especies fueron observadas en el tiempo. El potencial hídrico no difirió entre las especies, los valores obtenidos fluctuaron entre - 0,2 y -2,0 MPa. La distribución de los MP en los tejidos de las plantas decreció en el siguiente orden: raíz > hoja > tallo. Ce y en algunos casos $\mathrm{He}$, mostraron mayor capacidad de eliminación de MP. El factor de translocación (FT) fue bajo en estas especies. He fue la especie que mostró FT > 1 para $\mathrm{Pb}$ (II), Cr (total) y $\mathrm{Hg}$ (II) y 0.40.9 para Cd (II) y Cr (VI). Las plantas evaluadas demuestran la potencialidad para la fitorremediación de LX y todas ellas se pueden clasificar como acumuladores de estos MP.
\end{abstract}

Palabras claves: clorofila, fitorremediación, lixiviado de relleno sanitario, metales pesados, potencial hídrico.

\begin{abstract}
This study evaluated the effect of the different concentrations of heavy metals $(\mathrm{HM})\left(\mathrm{Hg}^{+2}, \mathrm{Cd}^{+2}, \mathrm{Cr}^{+6}, \mathrm{~Pb}^{+2}\right)$, on the physiological response and HM accumulation of three plant species: Colocasia esculenta (Ce), Heliconia psittacorum (He) y Gynerium sagittatum (Gs), planted in subsurface horizontal flow wetland for landfill leachate (LL) treatment. Factorial design was used with 16 bioreactors were operated in two experimental blocks. Chlorophyll, water potential and heavy metal content in plant tissues were measured. The results obtained showed significant differences $(p<0,05)$ between chlorophyll values and tested variables over time. The water potential did not differ between the three plant species, as all values were found in a range between -0.2 and $-2.0 \mathrm{MPa}$. The distribution of heavy metals in plant tissues decreased in the following order: root $>$ leaf $>$ stem. $\mathrm{Ce}$ and in some cases $\mathrm{He}$, showed higher removal capacity of HM. The translocation factor (TF) was lower. $\mathrm{He}$ was the specie that showed higher $\mathrm{TF}>1$ for $\mathrm{Pb}$ (II), $\mathrm{Cr}$ (total) and $\mathrm{Hg}$ (II) and 0.4-0.9 for $\mathrm{Cd}$ (II) y $\mathrm{Cr}$ (VI). The tested plants demonstrated that they are suitable for phytoremediation of LL and all of them can be categorized as HM accumulators.
\end{abstract}

Keywords: chlorophyll, heavy metals, landfill leachate, phytoremediation, water potential. 


\section{Introducción}

El lixiviado de relleno sanitario (LX) es un agua residual compleja producida por la degradacion química y biológica de la materia contenida en los residuos, así como por la infiltración de agua lluvia a través de los residuos sólidos. La presencia de agua permite una combinación de procesos físicos, químicos y microbiológicos para transferir contaminantes del residuo sólido en descomposición al líquido, resultando así la formación del lixiviado (Lei et al., 2007). La composición del LX generalmente comprende materia orgánica, nutrientes $(\mathrm{N}, \mathrm{P})$, metales pesados, compuestos recalcitrantes y otros xenobióticos (Renou et al., 2008). Los metales pesados (MP) a diferencia de los contaminantes orgánicos, son persistentes en la naturaleza, por lo tanto tienden a acumularse en distintos nichos de los ecosistemas, son bioacumulados y bioaumentados a medida que se avanza en los diferentes niveles de la red trófica. Estas sustancias son de reconocido impacto en el ambiente (agua, aire y suelo) y algunos de ellos como el $\mathrm{Cd}^{2+}$ y $\mathrm{Pb}^{2+}$ están asociados a malformaciones congénitas (Cuartas, 2010).

La biorremediacion de lixiviados de rellenos sanitarios a través de humedales construidos subsuperficiales de flujo horizontal (HCSFH) inició a comienzos de la década de los 90's. Gran parte de los estudios han estado centrados en la eliminación de los contaminates con mayor presencia en el LX, como lo son la Demanda Química de Oxigeno (DQO), Demanda Biológica de Oxigeno $\left(\mathrm{DBO}_{5}\right)$, Nitrógeno Amoniacal $\left(\mathrm{N}_{-} \mathrm{NH}_{4}+\right)$, Fosforo Total (P-total) y Sólidos Suspendidos Totales (SST) (Vymazal, 2009).

La fitoremediación se define como el uso de plantas para eliminar, destruir o transformar contaminantes del suelo, agua y aire. En este proceso, las plantas son seleccionadas principalmente por su potencial fisiológico, como en el caso de enzimas presentes para tolerar y asimilar sustancias tóxicas, por sus tasas de crecimiento, por la profundidad de sus raíces y su habilidad para bioacumular y/o degradar contaminantes (Panich-Patetal.,2010), mostrando que las plantas juegan un rol fundamental en los procesos de fitorremediación de aguas residuales. En este sentido, investigaciones encaminadas a encontrar nuevas especies tropicales son necesarias, debido a su importancia en el éxito de la fitorremediación.

La clorofila $(\mathrm{Cl})$ es uno de los principales indicadores de la capacidad fotosintética en plantas y la cuantificación de los pigmentos fotosintéticos contribuye a conocer mejor el comportamiento de las plantas durante su ciclo de desarrollo en presencia de metales pesados (Fortes et al., 2009). Por otro lado, el potencial hídrico $(\mathrm{Ph})$ foliar expresa el estado energético del agua en las células y en los tejidos vegetales y su determinación periódica, es un método alternativo para el conocimiento de la necesidad de agua de la planta durante el riego; así como para controlar la magnitud del estrés hídrico ya sea por exceso o defecto de agua (García et al., 2010). Por lo tanto, evaluar las respuestas fisiológicas de las plantas en los sistemas de humedales, es importante, no solo para determinar su capacidad de acumulación de metales, sino también para tener un conocimiento sobre el nivel de estrés que las plantas experimentan por los MP y su efecto en el crecimiento de las mismas.

El presente estudio evaluó el efecto en la respuesta fisiologica y la capacidad de acumulación de los $\mathrm{MP} \mathrm{Hg}^{+2}, \mathrm{Cd}^{+2}, \mathrm{Cr}^{+6} \mathrm{y} \mathrm{Pb}^{+2}$, en tres especies vegetales tropicales (G. sagittatum, C. esculenta, y H. psittacorum) sembradas en HCSFH a escala de microcosmos tratando LX sintético.

\section{Materiales y métodos}

\section{1 Área de estudio}

El experimento se desarrolló durante 60 días en la micro-estación de investigación de Biología, Universidad del Valle, campus Meléndez, CaliColombia (3o 22' 23.64" N; 76o 31'54.15” W) bajo condiciones de luz natural (radiación solar promedio $542 \mu \mathrm{mol} \mathrm{s}^{-1} \mathrm{~m}^{-2}$ ), fotoperiodo de 12:12 $\mathrm{hr}$, temperatura ambiental promedio de $27^{\circ} \mathrm{C}$, y $66 \%$ de humedad relativa promedio. 


\subsection{Diseño experimental y unidades experimentales}

El diseño experimental incluyó un diseño factorial con dos factores: i) Especie de plantas (tres especies) y ii) Concentración de MP en el LX (dos concentraciones). Los HCSFH se arreglaron en dos bloques experimentales ( $\mathrm{T} 1 \& \mathrm{~T} 2)$, acomodados aleatoriamente y operados en paralelo. Cada bloque consistió en seis reactores con plantas (tres reactores, una especie por reactor con respectivo duplicado) y dos unidades sin plantas, para un total de 8 reactores por bloque, 16 en total por los dos bloques experimentales. Así mismo, se contó con tres unidades de control (una especie por reactor) con su respectivo duplicado (seis reactores en total) alimentadas con agua potable y solución Hoagland. Los HCSFH fueron construidos en fibra de vidrio a escala de microcosmos con $0.60,0.30$ y $0.50 \mathrm{~m}$ de largo, ancho y alto respectivamente, con una lamina de agua de $0.40 \mathrm{~m}$ (Figura 1). La alimentación de los reactores fue a gravedad bajo la condición de flujo semi-continuo por ocho horas al día con una caudal de $0.010 \mathrm{~m}^{3} \mathrm{~d}^{-1}$, que equivale a un tiempo nominal de retención hidráulica (TRH) de tres días. El medio de soporte fue grava de 25 $\mathrm{mm}$ de diámetro y porosidad $(\eta)$ de $38.6 \%$, con una altura efectiva en el reactor de $0.45 \mathrm{~m}$.

\subsection{Especies vegetales}

Las tres especies tropicales seleccionadas para este estudio fueron Gynerium sagittatum (Gs),
Colocasia esculenta (Ce), y Heliconia psittacorum (He). Las plantas fueron obtenidas de un vivero local y sembradas en los humedales en edad juvenil (15 cm altura). La siembra se hizo por esquejes con tres individuos por reactor (densidad de plantas de 17 individuos por $\mathrm{m}^{2}$ ). Las especies fueron sembradas manualmente y colocadas en la grava a una profundidad similar.

\subsection{Lixiviado sintético}

Los reactores se alimentaron con lixiviado sintético, condición empleada en otros estudios que busca minimizar potenciales efectos inhibitorios en la fitoremediación (Nan et al., 2006; Liu et al., 2007). Los contaminantes incluidos en el LX fueron DQO, $\mathrm{DBO}_{5}, \mathrm{~N}\left(\mathrm{~N}-\mathrm{NTK}, \mathrm{N}_{-} \mathrm{NH}_{4}^{+}\right), \mathrm{PO}_{4}^{-}$ ${ }^{3}, \mathrm{Cd}$ (II), Hg (II), Pb (II) y Cr (VI). La DQO del LX consistió en ácidos grasos de cadena corta (acético: propiónico: butírico) en proporciones en volumen de 73\%: 23\%: 4\%, respectivamente (Moreno, 2009). $\mathrm{NH}_{4} \mathrm{Cl}$ fue empleado como fuente de amonio. Respecto a los MP, se emplearon sales de $\mathrm{HgCl}_{2}, \mathrm{PbSO}_{4}, \mathrm{~K}_{2} \mathrm{Cr}_{2} \mathrm{O}_{7}, \mathrm{y} \mathrm{CdCl}_{2}$. Todos los reactivos químicos fueron adquiridos a Merck, Alemania (99,99\% pureza). Las concentraciones en $\mu \mathrm{g} \mathrm{L}^{-1}$ de los MP utilizadas fueron en T1: 60.4; $283.8 ; 86.33 ; 1183.64$, y en T2: 71.56; 593.87; 253.6; 2016.45, para $\mathrm{Hg}$ (II), $\mathrm{Pb}$ (II), Cd (II), y Cr (VI), respectivamente. Cada solución fue preparada en soluciones stock de 1 litro de volumen de donde se tomaban porciones para la preparación diaria del LX sintético.
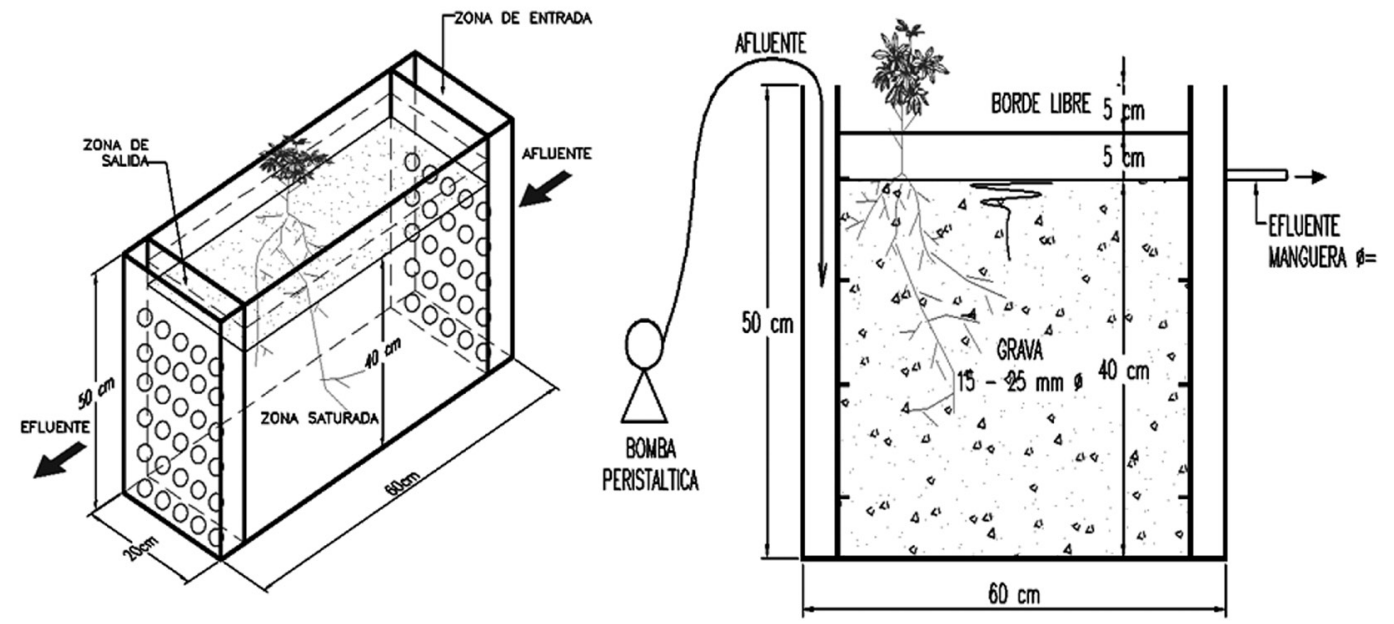

Figura 1. Esquema HCSFH utilizados en el estudio - Sin escala 
El LX fue preparado diariamente para evitar la descomposición de la fracción orgánica o la precipitación de algunas sales. En la Tabla 1 se presenta la característica promedio del LX empleado en el presente estudio y la calidad de un lixiviado real crudo (relleno sanitario de Presidente). Las unidades de control funcionaron bajo el mismo régimen de los reactores de los tratamientos pero alimentados con agua potable (sin cloro) y a cada reactor se le adicionaron 500 $\mathrm{ml}$ de solución Hoagland dos veces por semana, con la siguiente concentración de nutrientes en mg L ${ }^{-1}: 1200 \mathrm{Ca}\left(\mathrm{NO}_{3}\right)^{2}, 505 \mathrm{KNO}_{3}, 135 \mathrm{KH}_{2} \mathrm{PO}_{4}$, $988 \mathrm{MgSO}_{4}, 2.86 \mathrm{H}_{3} \mathrm{BO}_{3}, 1.81 \mathrm{MnCl}_{2} .4 \mathrm{H}_{2} \mathrm{O}$,
Al final del experimento las plantas fueron cosechadas de los HCSFH, lavadas con agua potable y enjuagadas con agua destilada. Todas las plantas fueron pesadas usando una balanza digital (FENIX, LEXUS electronics scale, \pm 0.1 g); luego, cortadas en tejido aéreo (tallo, rama, hojas) y terrestres (raíz) y pesados nuevamente. Posteriormente, las plantas se secaron a $80^{\circ} \mathrm{C}$ por 24 horas (Horno MLW Warmeschrank WS), cortadas y molidas (IKA 14 basic, hojas A11-2); luego a $0.5 \mathrm{~g}$ de muestra se le adicionaron $10 \mathrm{ml}$ de $\mathrm{HNO}_{3}$ (65\% volumen), como etapa previa a la digestión por microondas (CEM-Mars 5 X-press Duotemp, versión 194A07). Las concentraciones de Cd (II),

Tabla 1. Característica de LX sintético empleado en este estudio

\begin{tabular}{|c|c|c|c|c|c|c|c|c|c|}
\hline \multirow[t]{2}{*}{ Parámetro } & \multirow[t]{2}{*}{$N$} & \multicolumn{2}{|c|}{ Tratamiento 1} & \multicolumn{2}{|c|}{ Tratamiento 2} & \multicolumn{2}{|c|}{ Agua de Control } & \multicolumn{2}{|c|}{$\begin{array}{c}\text { Lixiviado Crudo } \\
\text { Presidente }+\end{array}$} \\
\hline & & Promedio & $S D$ & Promedio & $S D$ & Promedio & $S D$ & Promedio & $S D$ \\
\hline$D Q O(m g / L)$ & 8 & 735.5 & 143.6 & 735.5 & 143.6 & & & 6193.1 & 1889.7 \\
\hline$N T K(m g / L)$ & 8 & 132.4 & 46.4 & 132.4 & 46.4 & & & 2005.5 & 281.8 \\
\hline$N-N H 4(m g / L)$ & 8 & 99.4 & 28.2 & 99.4 & 28.2 & & & 1636 & 410.1 \\
\hline $\mathrm{N}-\mathrm{NO} 3(\mathrm{mg} / \mathrm{L})$ & 8 & 1.6 & 0.6 & 1.6 & 0.6 & & & 79.7 & 48 \\
\hline P-PO4 $(m g / L)$ & 6 & 7.3 & 3.4 & 7.3 & 3.4 & & & 46.4 & 20.6 \\
\hline$T(C)$ & 48 & 23.3 & 2.3 & 23.3 & 2.6 & 23.1 & 2.3 & 29 & 2 \\
\hline$p H$ & 48 & 5.3 & 0.9 & 5.3 & 0.9 & 6.8 & 0.9 & 8.1 & 0.2 \\
\hline$O D(m g / L)$ & 48 & 4.2 & 0.4 & 4.2 & 0.4 & 4.0 & 0.4 & 0.4 & 0.5 \\
\hline $\operatorname{Redox}(m V)$ & 47 & 222.5 & 134.8 & 224.8 & 130.6 & 251.6 & 132.6 & -19.5 & \\
\hline$C E(d S / m)$ & 48 & 1482.5 & 253.2 & 1512 & 223.6 & 145.1 & 22.1 & 24.3 & 3.4 \\
\hline Cr total $(\mu g / L)$ & 5 & 1644 & 352.9 & 2266 & 259.7 & - & - & - & - \\
\hline$C r(V I)(\mu g / L)$ & 5 & 1183.7 & 508.8 & 2016.3 & 672.1 & - & - & 5460 & 1651 \\
\hline$P b(I I)(\mu g / L)$ & 5 & 283.7 & 235.5 & 593.9 & 490.9 & - & - & 21.8 & 17.6 \\
\hline$C d(I I)(\mu g / L)$ & 5 & 86.3 & 48.4 & 253.6 & 12.4 & - & - & 4.2 & 3.2 \\
\hline$H g(I I)(\mu g / L)$ & 5 & 60.4 & 39.5 & 71.6 & 39.7 & - & - & 10.4 & 24 \\
\hline
\end{tabular}

$0.08 \quad \mathrm{CuSO}_{4} .5 \mathrm{H}_{2} \mathrm{O}, \quad 0.22 \quad \mathrm{ZnSO}_{4} .7 \mathrm{H}_{2} \mathrm{O}, \quad 0.11$ $\mathrm{Na}_{2} \mathrm{MoO}_{4} \cdot 2 \mathrm{H}_{2} \mathrm{O}, 3.00 \mathrm{FeSO}_{4} \cdot 7 \mathrm{H}_{2} \mathrm{O}$.

Las medición de las variables fisiológicas de potencial hídrico y clorofila se describen en la Tabla 2.
$\mathrm{Pb}$ (II), y Cr(total) fueron determinados a través de ICP-MS (Inductively Coupled Plasma - Mass Spectrometer; Thermo scientific Type: $X$-series $\left.2, \pm 1 \mu \mathrm{g} \mathrm{L}^{-1}\right)$. Hg (II) fue medido por absorción atómica con vapor frío (Shimadzu AA 6300, $\pm 1 \mu \mathrm{g}$

Tabla 2. Medición de parámetros fisiológicos en plantas

\begin{tabular}{cc}
\hline Parametro & Descripción \\
\hline Potencial & Se determinó en una hoja por individuo por especie por reactor, utilizando la cámara de \\
Hidrico & presión de Scholander (Modelo 1000). Medido una vez semanal \\
Clorofila & Se midió en tres hojas por individuo por especie por reactor, utilizando Clorofilometro \\
& (Minolta SPAD 502). La determinación se efectúo dos veces a la semana. \\
\hline
\end{tabular}


$\mathrm{L}^{-1}$ ) con generación de hidruros (Shimadzu HVG1). $\mathrm{Cr}$ (VI) fue determinado colorimétricamente usando un método espectrofotómetrico (Perkin Elmer, LAMBDA $20, \pm 2,8 \mu \mathrm{g} \mathrm{L}^{-1}$ ) a $540 \mathrm{~nm}$ de longitud de onda. Todas las determinaciones se realizaron acorde con APHA (2005). Muestras de estándares certificadas de lodo de aguas residuales de Holanda (No-143R. ID No 0811, May-042011), fueron empleadas como muestras de control de la calidad a los análisis a las muestras de plantas. Finalmente, mediciones de los MP a muestras de tejido de las especies vegetales antes de iniciar el estudio reportaron que todas las determinaciones estuvieron por debajo del límite de detección de los equipos y técnicas empleadas.

\subsection{Factor de trasladación (FT)}

El factor de translocación (FT) fue calculado dividiendo la concentración de metal acumulado en el tejido aéreo de la planta entre la concentración de metal acumulado en el tejido subterráneo de la planta; las concentraciones fueron reportadas en peso seco (PS) (Soda et al., 2012).

$$
F T=C_{A} / C_{u}
$$

Donde, CA es la concentración de metal en tejido aéreo de la planta ( $\mathrm{mg} \mathrm{kg}^{-1}$-PS) y $\mathrm{Cu}$ es la concentración de metal en tejido subterráneo de la planta ( $\mathrm{mg} \mathrm{kg}^{-1}$-PS). Grandes valores de FT ( $\left.>>1\right)$ implican una alta capacidad de translocación.

\subsection{Análisis de datos}

Las comparaciones entre el conjunto de datos obtenidos, la evaluación del efecto del tiempo en cada una de las variables estudiadas y el análisis estadístico de los resultados se realizó a través de la prueba de Anova de Medidas Repetidas con el software estadístico SPSS versión 19 (2010). El supuesto de esfericidad se determinó con un nivel de significancia del $5 \%(0.05)$. El estadístico $\mathrm{F}$ univariado fue utilizado mediante la aplicación del índice corrector épsilon al corregir los grados de libertad de F. El análisis se basó en los resultados obtenidos por las dos estimaciones de épsilon. Para el contraste de las pruebas de las hipótesis se utilizó una significancia del $5 \%$, con un nivel de confianza del $95 \%$, y se realizó la prueba de Tukey para evaluar la existencia de diferencias significativas entre los grupos de datos.

\section{Resultados y discusión}

La concentración de clorofila mostró diferencias entre las tres especies vegetales. $\mathrm{He}$ y $\mathrm{Ce}$ fueron las de mayor concentración de clorofila en los dos tratamiento y Gs obtuvo la menor producción (Figura 2). Los resultados obtenidos muestran que la producción de clorofila en las plantas expuestas a diferentes concentraciones de metales pesados fue distinta dependiendo de la especie vegetal y el tiempo, encontrándose diferencias significativas entre las especies y en el tiempo $(\mathrm{p}<0.05)$. La presencia de los MP en el medio, pudo activar el mecanismo de defensa de la planta ocasionando una mayor producción de enzimas con actividad peroxidasa, como por ejemplo, la ascorbato peroxidada, una enzima importante en los mecanismos antioxidantes de la planta (Conklin, 2001).

H. psittacorum presentó una mayor producción de clorofila en los tratamientos pero no superó a las plantas control, esta respuesta fisiológica pudo representar el máximo esfuerzo de esta especie para sobrevivir ante las condiciones de estrés a la cual fue sometida. No obstante, muchas plantas pueden crecer en ambientes contaminados ya que pueden desarrollar una variedad de mecanismos que minimizan el exceso de metales a los cuales están expuestas. Estos mecanismos incluyen: inmovilización de los iones tóxicos en la pared celular, impedimento de la absorción a través de las capas del protoplasma; inducción al estrés proteico como protección a la toxicidad metálica; compartamentalización y formación de compuestos con ácidos orgánicos e inorgánicos (Peña et al., 2005).

La síntesis de clorofila en $C$. esculenta durante los tratamientos fue superior a la de Gs e igual a la encontrada en las especies de control, condición que se presentó en el tratamiento T2. Esta respuesta fisiológica es probable que se haya dado por la activación de los mecanismos de reparación antes mencionados o por la activación de sistemas 


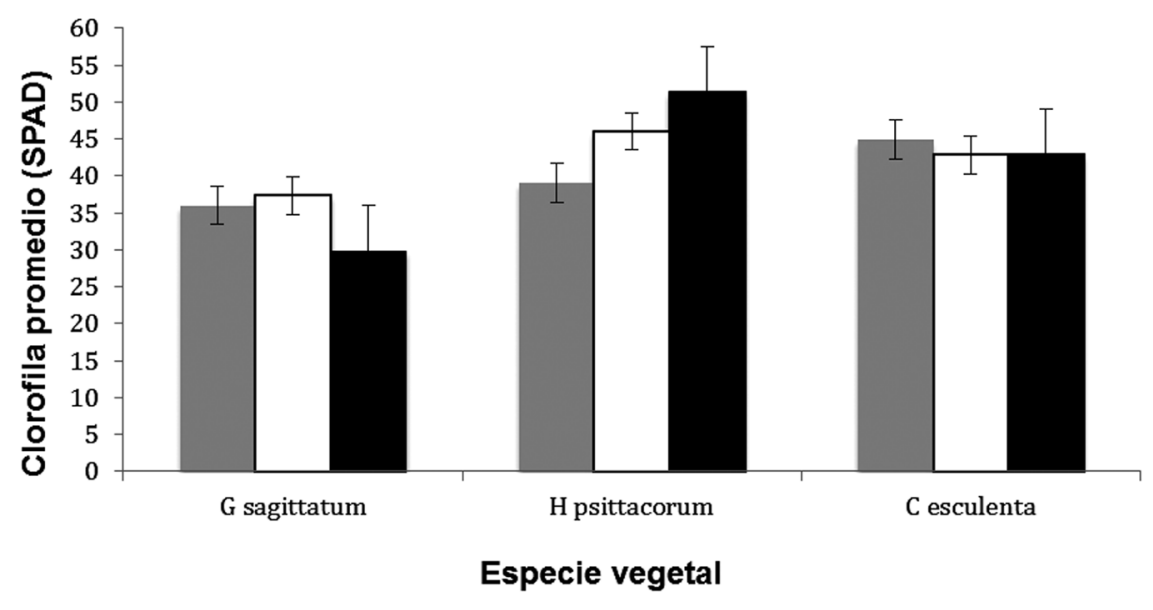

$\square \mathrm{T} 1 \square \mathrm{T} 2$ a Control

Figura 2. Valores promedio de clorofila en G. sagittatum, $H$. psittacorum y en C. esculenta

antioxidantes, compuestos por radicales libres de moléculas de barrido y las enzimas implicadas en el proceso de reparación generadas por el estrés metálico (Peña - Salamanca et al., 2011).

En el caso del potencial hidrico, no se presentaron diferencias significativas $(\mathrm{p}>0.05)$ para este parámetro entre las especies vegetales. Los resultados demuestran que la respuesta fisiológica respecto al estado hídrico de la planta no difirió entre las tres especies. De acuerdo a Zeiger y Taiz (2006) los resultados del potencial hídrico indican que las tres especies fueron sometidas a un estrés moderado, dado que los valores encontrados se hallaron en un rango entre $-0,2$ y $-1,2 \mathrm{MPa}$, en donde los procesos fisiológicos se pueden llevar a cabo en una relativa normalidad. En la Tabla 3 se observa que en el tratamiento T1, Gs y $C e$ presentaron un mejor comportamiento en el potencial hídrico respecto al control, mientras que en el tratamiento T2, Gs y He fueron las especies vegetales de mayor tolerancia frente al estrés hídrico con metales pesados.
Las especies vegetales del estudio experimentaron un estrés hídrico moderado, que involucra cambios rápidos y reversibles; por ejemplo, el ajuste osmótico, derivado de la acumulación de solutos (Zeiger y Taiz, 2006). En G. sagittatum, $H$. psittacorum y $C$. esculenta, es posible que las condiciones fisiológicas iníciales se puedan restablecer cuando el estrés deje de actuar, por tal razón estas especies vegetales pueden considerarse como plantas tolerantes al estrés hídrico generado en medios acuosos contaminados con metales pesados. De acuerdo a Olalla (2005) las especies que presentan estrategias de tolerancia al estrés hídrico registran mecanismos como el mantenimiento de turgencia celular (ajuste osmótico y ajuste elástico) y la tolerancia a la deshidratación (tolerancia protoplasmática).

El ajuste osmótico permite mantener la turgencia celular en condiciones de sequía, y con ello, los procesos relacionados como expansión y crecimiento celular, control de abertura estomática, fotosíntesis, entre otros. Este ajuste

Tabla 3. Valores del Potencial Hidrico (MPa) de las tres especies evaluadas bajo

las condiciones del estudio

\begin{tabular}{cccccccccc}
\hline \multirow{2}{*}{$\begin{array}{c}\text { Tiempo } \\
\text { (días) }\end{array}$} & \multicolumn{3}{c}{ Gynerum sagittatum } & \multicolumn{3}{c}{ Colocasia Esculenta } & \multicolumn{3}{c}{ Heliconia psittacorum } \\
\hline 0 & $\mathbf{T 1}$ & $\boldsymbol{T 2}$ & Control & $\boldsymbol{T 1}$ & $\boldsymbol{T 2}$ & Control & $\boldsymbol{T 1}$ & $\boldsymbol{T} 2$ & Control \\
\hline 10 & $-0,619$ & $-0,613$ & $-0,635$ & $-0,464$ & $-0,136$ & $-0,441$ & $-1,111$ & $-0,601$ & $-1,082$ \\
38 & $-0,361$ & $-0,704$ & $-0,734$ & $-0,390$ & $-0,345$ & $-0,345$ & $-1,02$ & $-1,069$ & $-0,771$ \\
50 & $-0,229$ & $-0,182$ & $-0,169$ & $-0,282$ & $-0,126$ & $-0,122$ & $-0,595$ & $-0,322$ & $-0,33$ \\
\hline
\end{tabular}


se consigue mediante la acumulación activa de solutos, fundamentalmente azucares solubles, aminoácidos (prolina y glicínbetaína) y en algunos casos potasio. Así, en las condiciones de estrés hídrico, las variedades presentan un mejor ajuste osmótico y una mejor extracción de agua, consiguiendo rendimientos mayores (Olalla, 2005), condición que se observó en las respuestas fisiológicas, respecto al estado hídrico en las plantas de este estudio.

Respecto a los MP, estos elementos en los tejidos de las plantas tendieron a decrecer en el siguiente orden: raíz $>$ hoja $>$ tallo. Sin embargo esta condición presentó variaciones dependiendo de la especie vegetal y de la concentración del metal. Por ejemplo, en $\mathrm{Ce}$ y en $\mathrm{He}$ el orden fue: raíz $>$ hoja > tallo, mientras que en $G s$, el orden para $\mathrm{Cr}$ (VI) y Hg (II) fue: hoja > raíz $>$ tallo.

La concentración de MP en raíces fue superior a la acumulada en los tejidos aéreos. La translocación del metal de la parte subterránea a la aérea difiere significativamente (Tabla 4). Ce presentó FTs $<1$ para casi todos los metales en los dos tratamientos, exceptuando $\mathrm{Cr}$ (VI) y $\mathrm{Pb}$ (II) en el tratamiento T2, mostrando que esta especie no distribuye en buena manera en sus tejidos el metal acumulado, dejando gran cantidad en la raíz; Cd (II) incluso no fue detectado en tejido aéreo en dos de las tres especies estudiadas, siendo G. Sagittatum la especie que logró acumular este metal en todos sus tejidos. Hg (II) fue el elemento con altos FTs (1.7) en Gs. He es la especie que mostró una mejor distribución de los metales en su tejidos, en el tratamiento T1, dos metales tuvieron FT > 1 y para los otros metales en los tratamientos T1 y T2 los valores fluctuaron entre 0.4 y 1.2. Los valores de FT variaron con los cambios de la concentración del metal en el LX, tal es el caso del $\mathrm{Hg}(\mathrm{II})$ que a mayor concentración de este metal en el LX G. sagittatum y $H$. psittacorum transfirieron más $\mathrm{Hg}^{+2}$ a las hojas y tallos, que la otra especie vegetal; el $\mathrm{Pb}^{+2}$ se transfirió en mayor medida a

Tabla 4. Concentración de MP $\left(\mathrm{mg} \mathrm{kg}^{-1}\right)$ y FT en las especies evaluadas

\begin{tabular}{|c|c|c|c|c|c|c|c|}
\hline Planta & Tratamiento & & $H g(I I)$ & Cd (II & $P b(I I)$ & $C r(V I)$ & Total-Cr \\
\hline \multirow{6}{*}{$\begin{array}{l}\text { Colocasia } \\
\text { esculenta }\end{array}$} & \multirow{3}{*}{$T 1$} & Raíz & $1,9 a$ & $7,3 a$ & $12,5 b$ & $N D$ & $112,6 a$ \\
\hline & & Aéreo & $0,8 a$ & $N D$ & $9,8 a$ & $4,6 a$ & $47,3 a$ \\
\hline & & $F T$ & $4 \times 10-1 e$ & - & $8 \times 10-1 e$ & - & $1 \times 10-1 e$ \\
\hline & \multirow{3}{*}{$T 2$} & Raíz & $2,6 a$ & $15,9 a$ & $20,6 \mathrm{~b}$ & $28,8 \mathrm{c}$ & $95,4 a$ \\
\hline & & Aéreo & $2,3 a$ & $N D$ & $23,2 a$ & $31,8 a$ & $25,7 a$ \\
\hline & & $F T$ & $9 \times 10-1 e$ & - & $1,1 \times 100 e$ & $1,1 \times 100 e$ & - \\
\hline \multirow{6}{*}{$\begin{array}{c}\text { Gynerum } \\
\text { sagittatum }\end{array}$} & \multirow{3}{*}{$T 1$} & Raiz & $1,5 a$ & $7,5 a$ & $9,3 b$ & $N D$ & $24,6 a$ \\
\hline & & Aéreo & $0,2 a$ & $3,9 a$ & $8,5 a$ & $3,5 a$ & $11,9 a$ \\
\hline & & $F T$ & $1 \times 10-1 e$ & $\begin{array}{c}6 \times 10-1 \\
e\end{array}$ & $9 \times 10-1 e$ & - & $4 \times 10-1 e$ \\
\hline & \multirow{3}{*}{$T 2$} & Raíz & $1,7 a$ & $28,2 a$ & $18,1 \mathrm{~b}$ & $0,4 c$ & $139,0 a$ \\
\hline & & Aéreo & $2,9 a$ & $6,0 a$ & $5,7 a$ & $N D$ & $15,8 a$ \\
\hline & & $F T$ & $\begin{array}{l}1,7 x \\
100 e\end{array}$ & $\begin{array}{c}2 \times 10-1 \\
e\end{array}$ & $3 \times 10-1 e$ & - & $1 \times 10-1 e$ \\
\hline \multirow{6}{*}{$\begin{array}{c}\text { Helicona } \\
\text { psittacorum }\end{array}$} & \multirow{3}{*}{$T 1$} & Raíz & $0,4 a$ & $2,3 a$ & $4,6 b$ & $1,7 c$ & $22,6 a$ \\
\hline & & Aéreo & $0,3 a$ & $N D$ & $6,1 a$ & $1,3 a$ & $27,3 a$ \\
\hline & & $F T$ & $9 \times 10-1 e$ & - & $\begin{array}{l}1,3 x \\
100 e\end{array}$ & $7 \times 10-1 e$ & $1,5 \times 100 \mathrm{e}$ \\
\hline & \multirow{3}{*}{$T 2$} & Raíz & $0,6 a$ & $5,0 a$ & $10,6 \mathrm{~b}$ & $7,6 c$ & $47,0 a$ \\
\hline & & Aéreo & $0,7 a$ & $N D$ & $7,1 a$ & $3,0 a$ & $16,5 a$ \\
\hline & & $F T$ & $\begin{array}{l}1,2 x \\
100 e\end{array}$ & - & $7 \times 10-1 e$ & $4 \times 10-1 e$ & $4 \times 10-1 e$ \\
\hline
\end{tabular}


las partes aéreas (hojas y tallos) de G. sagittatum y de $H$. psittacorum cuando la concentración del $\mathrm{Pb}^{+2}$ fue baja en el LX. Ninguna de las especies evaluadas revelo signos visible de clorosis al final del experimento (datos no mostrados).

Cd es más toxico que el $\mathrm{Pb}$ y el daño que este metal causa es más grande que el generado por el $\mathrm{Pb}(\mathrm{Niu}$ et al., 2007), afectando el crecimiento de la planta y su tolerancia a otros metales. En nuestro estudio, las tres especies vegetales acumularon más $\mathrm{Pb}$ que $\mathrm{Cd}$, esto sugiere un plausible mecanismo de defensa de la planta contra la toxicidad del $\mathrm{Cd}$.

Las especies evaluadas mostraron variaciones considerables en la toma y translocación de los metales a través de la fitoextracción (Tabla 4). Esta condición muestra que una apropiada selección de las especies puede ser crucial para mejorar la eficiencia de eliminación de MP en matrices liquidas a través de humedales construidos (Brisson y Chazarenc, 2009; Liu et al., 2010). Los resultados del estudio demuestran que las especies nativas pueden tolerar y acumular metales pesados como Cd (II), $\mathrm{Hg}$ (II), Cr (VI) y $\mathrm{Pb}$ (II), y mantienen una condición saludable (datos no mostrados). Esta característica es similar a los reportado en trabajos previos en humedales construidos (HC) con plantas emergentes como Phragmites australis, Arabis paniculata, Brasica juncea y Colocasia esculenta (Skinner et al., 2007; Moreno et al., 2008; Soda et al., 2012).\}

Bajo las condiciones del estudio, no se presentaron diferencias entre la concentración de MP en el agua y la acumulación de estos en el tejido subterráneo y aéreo de las plantas. Así mismo, no hubo diferencias significativas en los FTs (Tabla 4), sugiriendo que la concentración de cada MP tomado por la planta no interfiere con la transferencia de los otros tres MP en los tejidos de la especie vegetal.

\section{Conclusiones}

El comportamiento de la clorofila en las especies vegetales evaluadas se vio afectado por la concentración de metales pesados en el medio acuoso. C. esculenta y $H$. psittacorum fueron las especies vegetales de mayor concentración de clorofila durante el tiempo de experimentación para ambos tratamientos.

No se observaron diferencias significativas en el potencial hídrico entre las tres especies vegetales estudiadas, sin embargo C. esculenta resultó ser la especie vegetal con la mejor respuesta hídrica entre las especies, ya que los valores de este parametro fueron los más cercanos a cero en ambos tratamientos.

Las especies evaluadas no alcanzaron la concentración de metales en sus tejidos para ser catalogadas como hiperacumuladoras, pero mostraron un buen desempeño para ser clasificadas como acumuladoras de los metales pesados estudiados en este trabajo. G. sagittatum fue la especie con el mejor desempeño para los cuatro metales, seguido de H. psittacorum y C. esculenta respectivamente.

\section{Agradecimientos}

Los autores expresan sus agradecimientos al Instituto UNESCO-IHE-Insttitue for Water Education, el cual a través del programa PoWER-DUPC-UPaRF Categoria III, financiaron esta investigación.

\section{Referencias bibliográficas}

APHA. 2005. Standard Methods for the Examination of Water and Wastewater. 21st ed. Washington (DC): American Public Health Association.

Brisson, J, \& Chazarenc F. (2009). Maximizing pollutant removal in constructed wetlands: should we pay more attention to macrophyte species selection?. Journal Science Total Environment. 407, 3923-3930.

Conklin, PL., (2001). Recent advances in the role and biosynthesis of ascorbic acid in plants. Journal Plant Cell Environment. 24, 383 - 394.

Cuartas, D., Ariza, Y., Pachajoa, H,. \& Méndez F. (2011). Análisis de la distribución espacial y temporal de las anomalías congénitas registradas entre 2004 y 2008 en un Hospital de Tercer Nivel 
de la ciudad de Cali, Colombia. Colombia Médica. 42(1), 9-16.

Fortes, D., Herrera, H S., González, S., García, M., Romero, A., Cruz, A M., (2010). Comportamiento de los pigmentos fotosintéticos, según la edad de rebrote después del pastoreo de Pennisetum purpureum vc. Cuba CT-115 en la estación poco lluviosa. Revista Cubana de Ciencia Agrícola. 44 (4), 427-431.

García, A, Cunn, R, Montero, L., (2010). Efecto de la hora del día en el potencial hídrico foliar del sorgo y su relación con la humedad en el suelo. Rev Ciencia Técnologia Agricola. 19(3), 7 - 11.

Lei, Y, Shen, Z, Huang, R, Wang, W., (2007). Treatment of landfill leachate by combined agedrefuse bioreactor and electro-oxidation. Water Research. 41, 2417-2426.

Liu, J., Dong, Y., Wang, D., \& Xu, J., (2007). Accumulation of $\mathrm{Cd}, \mathrm{Pb}$ and $\mathrm{Zn}$ by 19 wetlands plant species in constructed wetland. Journal. Hazard Material. 147, 947-953.

Liu, J, Li, G, Shao, W, Xu, J, Wang, D., (2010). Variations in uptake and translocation of copper, Chromium and Nickel nineteen wetlands plant species. Pedosphere. 20, 96-103.

Moreno, CP. (2009). Factibilidad de la biodegradacion anaerobia de lixiviados en combinacion con agua residual domestica. BSc tesis. Escuela EIDENAR, Universidad del Valle, Cali, Colombia.

Moreno, F.N., Anderson, C.W.N., Stewart R.B., Robinson B.H., (2008). Phytofiltration of mercurycontaminated water: Volatilisation and plant accumulation aspects. Environmental Experimental Botany. 62, 78-85.

Nan, S., Li, G., \& Xin, X., (2006). Municipal landfill leachate induce cytogenetic damage in root tips of Hordeum vulgare. Ecotoxicology and Environmental safety, 63, 469-473.

Niu, Z., Sun, L., Sun, T., L, Y., Wang, H., (2007). Evaluation of phytoextracting cadmium and lead by sunflower, ricinus, alfalfa and mustard in hydroponic culture. Journal Environmental Science. 19, 961-967.

Olalla, FM., (2005). Agua y Agronomía. 1ed. Madrid: Editorial Mundi-Prensa Libros, S.A.

Panich-Pat, T., Upatham, S, Pokethitiyook, P, Kruatrachue M, Lanza GR., (2010). Phytoextraction of metal contaminants by Typha angustifolia: interaction of lead and cadmium in soil-water microcosms. Journal Environmental Protection Ecology. 1, 431-437.

Peña, EJ, Palacios, ML, Ospina ,N., (2005). Algas como indicadoras de contaminación. Colección Ciencia Físicas, Exactas y Naturales. 1ed. Cali: Universidad del Valle - Programa Editorial.

Peña-Salamanca, EJ, Rengifo-Gallego, AL, Benitez-Campo, N., (2011). Detoxification mechanisms of heavy metals by algal-bacteria consortia. En: Se-Kwon Kim editor. Handbook of marine macroalgae: biotechnology and applied phycology. Chichester: Wiley-Blackwell. 441-452.

Ramirez, F., (2014). Tratamiento primario de lixiviado intermedio del relleno sanitario mediante Bioreactor Laguna anaerobia de Alta Tasa (BLAAT®): Evaluacion del desempeño a escala piloto en ambiente tropical. BSc (Tesis), Programa Academico de Ingenieria Sanitaria y Ambiental, Universidad del Valle, Cali, Colombia.

Renou, S, Givaudan, JG, Poulain, S, Dirassouyan, F, Moulin, P., (2008). Landfill leachate treatment: review and opportunity. Journal Hazard Material. $150,468-493$.

Skinner, K., Wright, N., Porter-Goff,, E., (2007). Mercury uptake and accumulation by four species of aquatic plants. Environmental. Pollution. 145, 234-237.

Soda, S., Hamada, T., Yamoaka, Y., Ike, M., Nakazato, H., Saeki, Y., Kasamatsu, T., Sakurai, Y., (2012). Constructed wetlands for advanced treatment of wastewater with a complex matrix from a metal-processing plant: Bioconcentration 
and translocation factors of various metals in Acorus gramineus and Cyperus alternifolius. Ecologycal engineering. 39, 63-70.

Vymazal, J., (2009). The use of constructed wetlands with horizontal sub-surface flow for various types of wastewater. Ecological Engineering. 35, 1-17.

Zeiger, E, Taiz, L., (2006). Fisiología vegetal. 3 ed. Castellon:Madrid. Universitat Jaume I. 